\title{
Exocytosis, Endocytosis, and Their Coupling in Excitable Cells
}

\author{
Kuo Liang ${ }^{1 \dagger}$, Lisi Wei ${ }^{2 \dagger}$ and Liangyi Chen ${ }^{2 *}$ \\ ${ }^{1}$ Department of General Surgery, XuanWu Hospital, Capital Medical University, Beijing, China, ${ }^{2}$ State Key Laboratory of \\ Membrane Biology, Beijing Key Laboratory of Cardiometabolic Molecular Medicine, Institute of Molecular Medicine, Peking \\ University, Beijing, China
}

Evoked exocytosis in excitable cells is fast and spatially confined and must be followed by coupled endocytosis to enable sustained exocytosis while maintaining the balance of the vesicle pool and the plasma membrane. Various types of exocytosis and endocytosis exist in these excitable cells, as those has been found from different types of experiments conducted in different cell types. Correlating these diversified types of exocytosis and endocytosis is problematic. By providing an outline of different exocytosis and endocytosis processes and possible coupling mechanisms here, we emphasize that the endocytic pathway may be pre-determined at the time the vesicle chooses to fuse with the plasma membrane in one specific mode. Therefore, understanding the early intermediate stages of vesicle exocytosis may be instrumental in exploring the mechanism of tailing endocytosis.

\section{OPEN ACCESS}

Edited by:

Cong Ma,

Huazhong University of Science and

Technology, China

Reviewed by:

Qiangjun Zhou,

Howard Hughes Medical Institute,

USA

Xuelin LOU,

University of Wisconsin-Madison, USA

*Correspondence:

Liangyi Chen

lychen@pku.edu.cn

${ }^{\dagger}$ Co-first authors.

Received: 18 January 2017 Accepted: 31 March 2017 Published: 19 April 2017

Citation:

Liang K, Wei L and Chen L (2017)

Exocytosis, Endocytosis, and Their

Coupling in Excitable Cells.

Front. Mol. Neurosci. 10:109.

doi: 10.3389/fnmol.2017.00109
Keywords: exocytosis, endocytosis, kiss and run, kiss and stay, compound fusion, multivesicular exocytosis, clathrin

\section{INTRODUCTION}

Vesicle exocytosis is a fundamental cellular process that regulates many biological events, such as the release of neurotransmitters, hormones, and cytokines and delivery of proteins and lipids to the plasma membrane for cell repair, growth, migration, and regulation of cell signaling (Alabi and Tsien, 2013; Wu L. G. et al., 2014). In excitable cells, such as neurons and endocrine cells, regulated exocytosis is triggered within milliseconds after membrane depolarization. Upon strong stimulation, a massive fusion of secretory vesicles could occur at designated release sites within a short period of time. Therefore, compared with constitutive exocytosis in non-excitable cells, regulated exocytosis must be equipped with specialized machinery that enables fast, $\mathrm{Ca}^{2+}$ dependent, spatially defined exocytosis. Tailing endocytosis must match with exocytosis to recycle exocytosed vesicular components and clear release sites on the plasma membrane in a timely fashion. Based on the kinetics, structures, and molecules involved in different cell types, a variety of exocytosis and endocytosis subtypes have been proposed. However, how these mechanisms are coupled in space and time remains mysterious. Here, we have provided an outline of different exocytic and endocytic processes and how they may be coupled by different factors.

\section{EXOCYTOSIS IN EXCITABLE CELLS}

Exocytosis requires a merging of the vesicular membrane into the plasma membrane. Through shielding of the negative charge on the bilayer surface, diminishing electrostatic repulsion force, and overcoming the dehydration barrier, two bilayers can merge into one. Formation of an assembled ternary SNARE complex provides the required energy. Depending on the fate of the vesicular 
components upon lipid merging, exocytosis can progress by fully inter-mixing the vesicular membrane components with the plasma membrane (full fusion), fusing with the plasma membrane via a transient flickering of the fusion pore (kiss and run; An and Zenisek, 2004; Rizzoli and Jahn, 2007; Alabi and Tsien, 2013), or fusing with a partially retained vesicular membrane structure and components at the exocytic site (kiss and stay; Taraska et al., 2003; An and Zenisek, 2004; Tsuboi et al., 2004; Rizzoli and Jahn, 2007).

\section{Different Models of Fusion Pore Formation}

Intrinsically, fusion machinery must operate with the formation and the expansion of an omega-shaped pore structure, which minimizes leakage from the vesicle and cytosol during exocytosis. Such a process requires coordinated distortion and controlled disruption of two lipid bilayers to form a water-filling fusion pore, which cannot be observed (van den Bogaart et al., 2010) directly in vivo due to its small size and short lifetime (Lindau and Alvarez de Toledo, 2003). Electrophysiological methods, on the other hand, provide a brief glimpse of some pore intermediates. Based on these indirect estimations, there exists three models that describe the fusion pore, a lipidic (Chanturiya et al., 1997) or a proteinaceous (Han et al., 2004) pore or a hybrid of the lipid and protein composition (Bao et al., 2016; Sharma and Lindau, 2016). For a lipidic pore, fusion starts with protrusion of two bilayers toward each other in a very narrow region, followed by the merge of the two proximal monolayers of each bilayer (stalk), the enlargement of the merged region to form one bilayer (hemifusion), and the final formation of a lipidic fusion pore. Formation of the stalk and hemifusion diagram ensures the expansion of the pore without a leak. Such a fusion pore does not require multiple copies of SNARE complexes. Instead, one pair of SNARE proteins, firmly anchored on the vesicular and plasma membrane with transmembrane (TM) segments, may interact with each other to provide the force to pull the different membranes together (van den Bogaart et al., 2010).

In 1987, Almers and co-workers measured the initial pore conductance during exocytosis of mast cells to be 200-300 pS, equivalent to a pore of diameter of $\sim 2 \mathrm{~nm}$ (Breckenridge and Almers, 1987). This value is similar to the conductance of $\mathrm{K}$ channel, inspiring the early hypothesis that the fusion pore is a proteinaceous gap junction channel. In 2004, using tryptophan scanning mutagenesis of the syntaxin TM anchor, Han et al. identified three critical residues that reduced the amplitude of the foot signal of an amperometry recording (Han et al., 2004). These positions are positioned along one face of the alpha-helix, promoting the idea that they might face the inside of a pore. Based on these results, they proposed a provocative hypothesis that the TM domains of 6-8 syntaxin molecules are arranged in a ring to form one half of a gap-junction-like pore, with the other half formed by the TM domains of synaptobrevin (Syb2). After the formation of the protein-lined pore, the pore could close again ("kiss and run"; Albillos et al., 1997; MacDonald et al., 2006) or allow the membrane lipids to enter to facilitate pore expansion and complete membrane merge ("pore dilation" or "full fusion"). This interpretation is supported by the existence of syntaxin clusters on the plasma membrane of endocrine and synapses (Barg et al., 2010; van den Bogaart et al., 2011), as well as three or more copies of SNARE proteins that are required for the fast release of secretory vesicles (Domanska et al., 2009; Mohrmann et al., 2010). This model, however, requires fusogenic proteins to be in perfect alignment to constrict lipid flow during the initial pore opening, which have not been proved experimentally. Changes in the amplitude of foot signals could be due to different extents of vesicular filling (Sombers et al., 2004) and different dissociation kinetics of neurotransmitters from the vesicular matrix (Reigada et al., 2003). The impact of a syntaxin mutation on the release kinetics thus may provide an alternative explanation. Other amperometric investigations also reported controversial results, such as very large fluctuations of foot signals characteristic of variable and lipidic pores.

A combination of these two models yielded a model with a pore that is both lipidic and proteinaceous. Recently, two Syb2 molecules have been shown to be able to be incorporated within a nanodisc with a diameter of $6 \mathrm{~nm}$, which readily fuses with t-SNARE-containing vesicles and can be blocked by mutations of critical residues in the TM domain of Syb2. Given that such a small nanodisc appeared to be too small to accommodate a lipidic pore, and at least three TM domains are required to line up a proteinaceous pore, these results suggest that the pore itself must be a hybrid of both proteins and lipids (Bao et al., 2016). According to the molecular dynamic simulation, the water-filled fusion pore traversing the membrane and the nanodisc constitutes both the lipid head group and the c-termini of the TM domains of Syb2 and syntaxin (Sharma and Lindau, 2016). Whether such a hybrid model works in real cells remains to be determined.

\section{Pore Opening and Full Fusion}

Under electron microscopy (EM), the earliest seen fusion pores of synaptic vesicles were never $<20 \mathrm{~nm}$ despite the observed 3-4 ms after a single stimulus (Heuser and Reese, 1981) and were mostly $\sim 150 \mathrm{~nm}$ for dense-core vesicle exocytosis in Limulus amebocytes (Ornberg and Reese, 1981). In contrast, using cell-attached membrane capacitance recording, fusion pore conductance of secretory vesicles range from 30 to $1,000 \mathrm{pS}$ (Breckenridge and Almers, 1987; Lindau and Alvarez de Toledo, 2003; He et al., 2006; MacDonald et al., 2006), corresponding to a fusion pore diameter of 1-7 $\mathrm{nm}$. Fusion pores larger than $10 \mathrm{~nm}$, as those observed under EM, will result in pore conductance approaching infinity, rendering estimation of pore size impossible. Additionally, these large fusion intermediates do not restrict the diffusion of neurotransmitters and small neuropeptides such as neuropeptide-Y (NPY) (Tsuboi et al., 2004). Thus, they are undetectable with either amperometry or membrane capacitance recordings. On the other hand, both electrophysiological methods provide an estimation of the pore duration before final dilation to be $\sim 10-80 \mathrm{~ms}$ in endocrine cells on average. Therefore, it is intriguing that a small pore $<10 \mathrm{~nm}$ was never observed under EM, provided that ultrafast-freezing EM should have sufficient temporal and spatial resolution in principal. Whether the small fusion pore intermediate is a rare event compared to other fusion intermediates or ultrafastfreezing EM lacks the resolution and contrast to resolve such 
small pores remains unknown. Live cell fluorescence microscopy, including super-resolution microscopy (Huang et al., 2009; Schermelleh et al., 2010), does not have the sufficient spatial and temporal resolution to observe small fusion pores either.

The large fusion pore observed, on the other hand, may represent an intermediate before full collapse of vesicles. A secretory vesicle contains $60-70$ copies of Syb2, of which 1-3 are used during the fusion process. All of these Syb2 molecules, as well as other vesicular proteins such as synaptotagmin, are completely lost on the plasma membrane during the full collapse of the vesicle. Clathrin-mediated endocytosis must be initiated to collect and precisely recycle these vesicular membrane components rapidly. Originally believed to be a slow process, we find that the migration of preformed clathrin-mediate pits (CCPs) on the plasma membrane to the vesicle release sites is key to the clearance of exocytic slots in a timely fashion (Figure 1; Yuan et al., 2015), which may explain the fast clathrinmediated endocytosis observed in neurons (time constant of 3-10 s; Granseth et al., 2006; Zhu et al., 2009).

\section{Kiss and Run (KR)}

Resealing of a small fusion pore leads to a KR event, which is both an exocytic and an endocytic process. KR events are detected as the "stand-alone" foot signals in amperometry recordings (Zhou and Misler, 1996; Albillos et al., 1997) or membrane capacitance flickers in capacitance recordings (Albillos et al., 1997; He et al., 2006; MacDonald et al., 2006). However, electrophysiology methods cannot identify the integrity of the vesicular shape and composition after a vesicle performs a KR event. KR was originally defined by Ceccarelli et al. (1973) under EM as the fusion of vesicles with preservation of vesicle morphology. Therefore, the conservation of the vesicle shape, as observed with EM and live cell fluorescence microscopy, along with a small pore probed with electrophysiology technologies are cornerstone features of KR (Alabi and Tsien, 2013).

Various conditions have been shown to promote KR in a number of secretory cells, including high cytosolic $\mathrm{Ca}^{2+}$ (Ales et al., 1999) and activation of PKA (MacDonald et al., 2006). Despite this knowledge, the mechanisms of the resealing and flickering of a fusion pore remain elusive. In the lipidic pore model, each intermediate structure is at its free energy minima, and an injection of exogenous energy is needed for the transition between different fusion intermediates. Therefore, fusing vesicles remain connected to the plasma membrane with a narrow pore until the addition of new proteins and lipids to the fusion machinery reverses the process. Indeed, a variety of proteins, such as dynamin (Anantharam et al., 2011; Jackson et al., 2015), myosin II, actin (Aoki et al., 2010), SNARE proteins (Fang et al., 2008; Gucek et al., 2016), synaptotagmin (Wang et al., 2001; Lai et al., 2013), and complexin (Dhara et al., 2014) have been found to affect the fusion pore dynamics in chromaffin and PC12 cells. These studies highlight an active role of these components in impacting the fusion pore. Alternatively, it is hypothesized that the release of energy associated with the formation of one SNARE bundle is insufficient to overcome the restraining force from the intact vesicle-vesicle and vesiclecytoskeleton filamentous web that opposes full vesicle collapse
(Alabi and Tsien, 2013). Therefore, upon completion of the transSNARE complex formation and diminishing of the countering force against pore constriction, the vesicle pore reseals to be intact again. The difference between these two models is that the force opposing fusion dilation is constitutively present at the release sites in the latter model, therefore bypassing the need for acute and coordinated recruitment of facilitating membrane components.

The physiological significance of KR in endocrine cells has been well-established. In pancreatic $\beta$-cells, the KR of large densecore vesicles (LDCV) and small vesicles allows for the selective release of ATP and GABA, respectively. In contrast, insulin crystals within LDCVs are retained within the lumen during the transient flickering of fusion pores (MacDonald et al., 2006). The release of peptides from other endocrine cells is also likely to be limited, since the transient brightening with no diffusion of fluorescent-tagged NPY puncta is regarded as a KR event under total internal reflection fluorescence (TIRF) microscopy (Tsuboi and Rutter, 2003). KR is also identified in the fusion of synaptic vesicles in synapses (He et al., 2006; Zhang et al., 2009), which may add another layer of post-fusional regulation and enables non-quantal synaptic transmission in principal. However, even for the smallest fusion pore opening, the vesicle will be drained of transmitter within tens of milliseconds, long before the fusion pore closes. Therefore, the KR model is unlikely to regulate vesicle release post-fusionally.

Alternatively, KR may confer an ultrafast and efficient recycling process independent of clathrin (He et al., 2006; Zhang et al., 2009). A KR event will lead to a fast and efficient recycling of almost all vesicular components, as well as immediate on-site refilling of neurotransmitters. The same vesicle then can fuse multiple times (Zhang et al., 2009), while the previously used cis-SNARE complex in the previous round of fusion needs to be removed from the vesicle to prevent blockade of the second round of fusion. However, it is unclear how such a cis-SNARE complex passes through the small flickering pore and diffuses into the plasma membrane without affecting the pore, given that the SNARE protein could be part of the pore itself. Nevertheless, by expelling a few Syb2 molecules used for each round of fusion, a KR event is more efficient in maintaining the identity of the vesicle than discharging all Syb2 molecules upon every instance of vesicle exocytosis. By keeping the vesicular V-ATPase, resealed vesicles can gradually re-acidify, which permits $\mathrm{pH}$ gradientcoupled refilling of the vesicle with neurotransmitters (Alabi and Tsien, 2013). In theory, this process may promote rapid recovery of neurotransmission during sequential stimulations. However, this hypothetical benefit is in disagreement with the experimental data that KR is prevalent at the beginning of action potential trains but is eventually replaced by full fusion upon sustained firing in hippocampal neurons (Zhang et al., 2009). Therefore, the physiological significance of KR in synapses remains unknown.

\section{Kiss and Stay (KS)}

In endocrine cells, in addition to probing fusion pores indirectly with electrophysiological methods, imaging technologies such as spinning disc confocal and TIRF microscopy provide the spatiotemporal correlation of fusion events with concurrent 

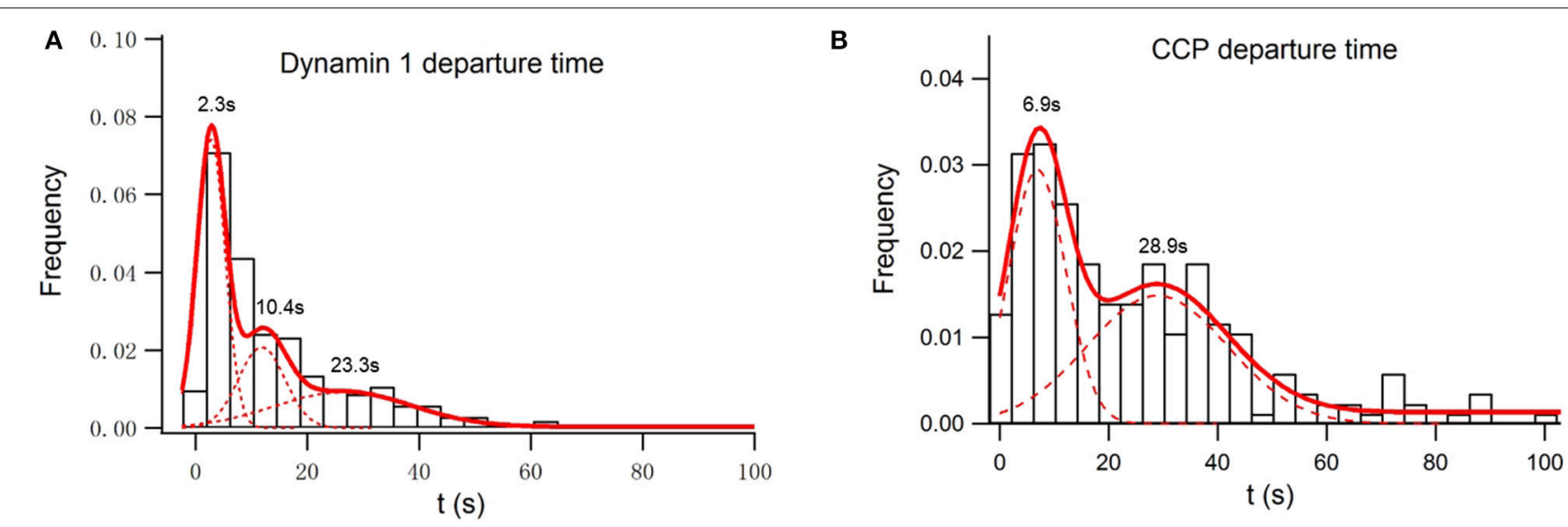

C
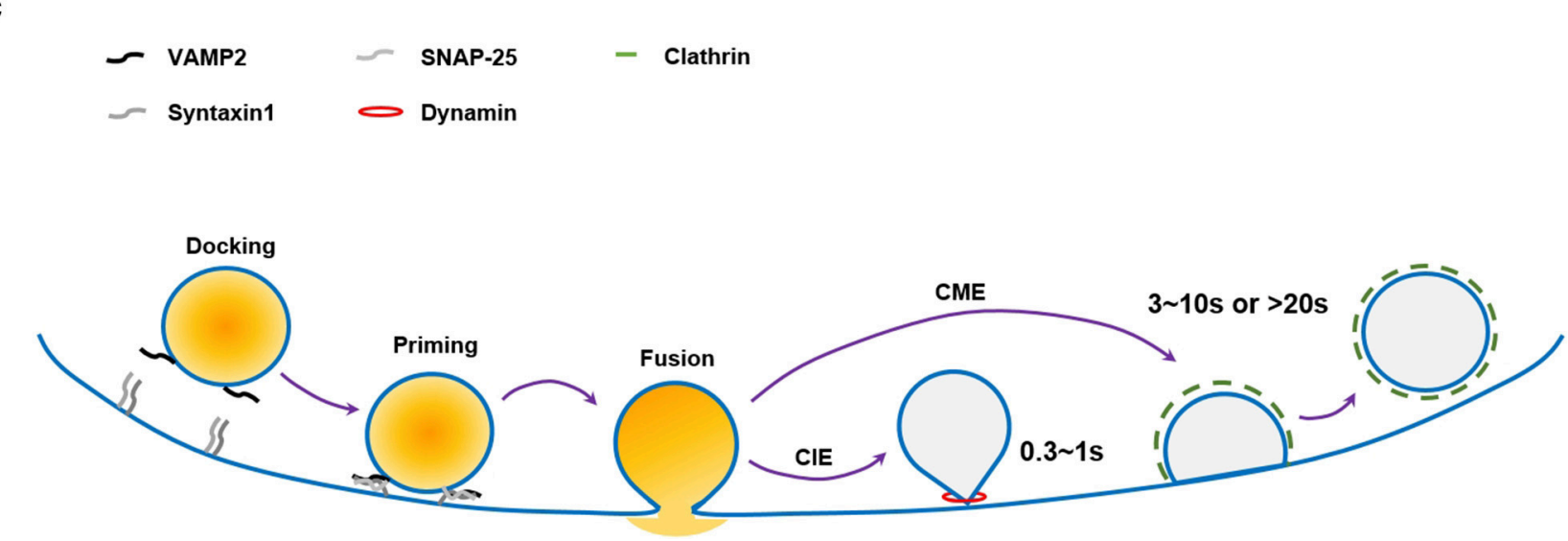

FIGURE 1 | Simulation of membrane capacitance decay at a fusion site due to clathrin-dependent endocytosis or both clathrin-dependent and -independent endocytosis. INS-1 cells were transfected with VAMP2-pHluorin and clathrin-DsRed and were stimulated with $70 \mathrm{mM} \mathrm{KCl}$ and $15 \mathrm{mM}$ glucose. Overall, $55 \pm 3 \%$ of vesicle fusion events were associated with on-site recruitment of dynamin 1. (A) Normalized histogram of departure times of recruited Dyn1 puncta at fusion sites. It can be fitted with a three-component Gaussian distribution centered at $2.3 \pm 0.11,10.4 \pm 0.5$, and $23.3 \pm 3.7 \mathrm{~s}$ and contributing to $\sim 39 \pm 3,24 \pm 6$, and $34 \pm 8 \%$ of the total population, respectively $(n=246)$. The latter two populations were dependent on clathrin, while the first one was independent of clathrin (Figure S2 in He et al., 2009). (B) Normalized histogram of departure times of recruited Clathrin puncta at fusion sites. It can be fitted with a two-component Gaussian distribution centered at $6.9 \pm 0.5$ and $28.9 \pm 2.6 \mathrm{~s}$ and contributing to $\sim 50 \pm 4$ and $44 \pm 6 \%$ of the total population, respectively $(n=215)$. (C) A scheme of how clathrin-dependent and independent endocytosis are differently coupled to exocytosis.

diffusion of vesicular lipids and proteins (Holroyd et al., 2002; Taraska et al., 2003; Tsuboi and Rutter, 2003; Tsuboi et al., 2004). In parallel to the small fusion pores detected from electrophysiological data, imaging reveals retention of vesicle membrane shape and some vesicle compositions after the exocytosis of LDCVs (Holroyd et al., 2002; Taraska et al., 2003; Tsuboi et al., 2004). In contrast to the retention of the majority of composition after a vesicle performs KR, the loss of vesicular lipids and the majority of some vesicular proteins such as Syb2 is obvious (Taraska and Almers, 2004; Tsuboi et al., 2004). Named as $\mathrm{KS}$ (or cavicapture), this process is regarded as an allosteric form of KR, sharing similar characteristics such as on-site recycling of vesicular components and its dependence on dynamin (An and Zenisek, 2004). In this sense, it is often regarded as a fast, clathrinindependent endocytosis that occurs at the fusion sites (Holroyd et al., 2002; Taraska et al., 2003; Tsuboi et al., 2004). However, we have shown that clathrin-dependent endocytosis could rapidly synchronized to occur at the fusion sites (Yuan et al., 2015), highlighting a necessity of classifying the identity of endocytosis based on molecules, rather than on kinetics and localization.

Moreover, it is unclear whether KS events identified by different fluorescence probes represent the same or different fusion intermediate stages. For example, discharge of NPY is much faster than that of fluorescent-tagged tissue plasminogen activator (tPA) in adrenal chromaffin cells and pancreatic $\beta$-cells (Tsuboi et al., 2004; Weiss et al., 2014). These data were initially interpreted to suggest that large tPAs $(\sim 10 \mathrm{~nm}$ in diameter) are prevented from free diffusion by the fusion pore, which do not interfere with diffusion of small lumen contents such as NPY ( $\sim 3 \mathrm{~nm}$ in diameter). However, overexpressed tPA changes the lumen composition of LDCVs, binds to the exposed luminal surface of fused chromaffin granules and slows down the release kinetics as measured by amperometry recordings (Weiss et al., 2014). A pore as large as $10 \mathrm{~nm}$ barely constrains 
diffusion of small neurotransmitters from vesicle lumen upon exocytosis. Alternatively, KS may represent a fusion intermediate later than the KR, which explains why it shares many mechanistic characteristic with the later. Given the prevalence of $\mathrm{KS}$ in endocrine cells, we speculate that the $20-150 \mathrm{~nm}$ fusion pores observed under EM may be in the KS state, although future direct proof is still needed.

Clearly, resolving fusion-associated membrane shape retention and dispersion of vesicular membrane during synaptic transmission is difficult due to the small sizes of synaptic vesicles and boutons. However, coupled and recycled vesicular membrane proteins are distinct from those left on the presynaptic membrane after exocytosis (Wienisch and Klingauf, 2006), highlighting a possible KS mechanism operating in synapses as well.

\section{Sequential Fusion and Multivesicular Exocytosis}

After a KS fusion event, the invaginated fusion site before endocytosis can be targeted to harbor the next rounds of exocytosis ("sequential fusion"; Takahashi et al., 2004; Kishimoto et al., 2005), creating deep invaginations on the plasma membrane that may resulted in internalization of one large endocytic vesicle ("bulk endocytosis"; $\mathrm{Wu}$ and $\mathrm{Wu}, 2007$; Wen et al., 2012). These invaginations were initially found in non-excitable cells such as pancreatic acinar cells, mast cells, eosinophils and neutrophils (Pickett and Edwardson, 2006) and were later discovered in excitable cells such as pancreatic $\beta$-cells and neurons (Kwan and Gaisano, 2005; He et al., 2009). During sequential fusion, cis-SNARE complexes need to be removed from the fusion sites, and new trans-SNAREs on the plasma membrane must diffuse into these invagination structures. By adopting this configuration, vesicles that exist deep within the cytosol readily fuse with the plasma membrane to release their contents. This configuration also creates spatially preferred sites on the plasma membrane, conferring a mechanism for generating exocytosis "hot spots" in non-neuronal cells.

Multivesicular exocytosis is another form of exocytosis where vesicles fuse homotypically before interacting with the plasma membrane. In contrast to compound fusion, a multivesicular exocytosis event leads to a capacitance increase that is several folds higher than that caused by the fusion of a single vesicle (He et al., 2009). However, multivesicular exocytosis is rare, precluding it from being systematically and statistically analyzed. Therefore, whether these large capacitance jumps represent a distribution different from that represented by a fusion of a single vesicle or the long-tail region of one unified distribution remains to be determined. Under EM, multiple vesicles that are connected to each other but not with the plasma membrane are sometime observed, which is also taken as evidence supporting multivesicular exocytosis (Wu L. G. et al., 2014). However, these structures could also be due to the sequential fusion of vesicles to the invaginated site that exhibited pore closure, which has a distinct molecular mechanism. A multivesicular exocytosis event needs homotypical vesicle-vesicle fusion, which presumably uses different sets of SNAREs other than those used for vesicle-plasma membrane exocytosis, similar to what has been proposed for compound fusion (Thorn and Gaisano, 2012). In principal, compared to the fusion of multiple vesicles at one designated site for several rounds, a multivesicular fusion event will be more efficient in emptying vesicular contents within a short period of time given that fusion sites are limited. However, how multivesicular fusion operates in vivo remains elusive.

\section{COUPLED ENDOCYTOSIS IN EXCITABLE CELLS}

Unlike constitutive endocytosis in non-excitable cells, coupled endocytosis following evoked exocytosis must be fast and spatially matched with exocytosis to maintain the balance of surface membrane and the finite size of the readily releasable pool of vesicles. Kinetically, evoked endocytosis often consists of two phases, a fast endocytosis followed by a slow one (Artalejo et al., 2002; He et al., 2008; Lou et al., 2008; Wu et al., 2009). Mechanistically, the fast endocytosis is often regarded as clathrinindependent, while the slow one is often dependent on clathrin (He et al., 2008; Lou et al., 2008). Based on these kinetic and mechanical characteristics, a full collapsing of vesicle fusion is often thought to be associated with the slow, clathrin-dependent endocytosis, while the resealing of a fusion pore during a KR or KS is regarded as the fast mechanism underlying the coupled clathrin-independent endocytosis.

\section{Clathrin-Dependent Endocytosis}

With immunostaining and confocal microscopy, active zones have been found to be surrounded by a peri-active zone enriched with endocytic proteins such as clathrin and dynamin, which mediate clathrin-mediated endocytosis following synaptic transmission (Cano and Tabares, 2016). Using TIRF microscopy, exocytosis in MIN6 cells was found to be associated with on-site recruitment of the endocytic protein dynamin but not clathrin, epsin, or amphiphysin. These data were interpreted to suggest that only clathrin-independent endocytosis, a form of $\mathrm{KS}$, is spatially coupled to exocytosis in insulin-secreting $\beta$-cells (Tsuboi et al., 2004). However, do these spatially confined dynamin recruitments represent bona fide endocytosis? If they indeed represent clathrin-independent endocytosis, do their kinetics match with electrophysiological data? What is their physiological significance? These are questions left unexplored. Recently, we have systematically examined the exocytosis-endocytosis coupling in insulin-secreting cells (Yuan et al., 2015). We have revealed that clathrin can be recruited to the fusion sites in a fast and a slow manner, which were accompanied with the simultaneous recruitment of dynamin (Figures 1A,B refer to Figure S2 and Figure 1C in Yuan et al., 2015). The slow recruitment represents a de novo formation of clathrin-coated pits (CCPs), while the fast recruitment originates from preformed CCPs stably docked at the fusion sites or rapid movement of CCPs toward fusion sites on the plasma membrane. These spatially confined clathrin recruitments are indeed mediators of the endocytosis of vesicular proteins such as synaptotagmin VII and Syb2 (Yuan et al., 2015). Therefore, clathrin-dependent 
endocytosis can operate both at a fast and a slow pace, in agreement with similar findings in hippocampus neurons (Granseth et al., 2006; Zhu et al., 2009). We argue that the speed of designated endocytosis depends on the extent of synchronization of individual events, which cannot be used as the sole criteria for distinguishing clathrin-dependent from clathrin-independent endocytosis. As we have shown, the physiological significance of fast, clathrin-dependent endocytosis is also critical for sustained exocytosis during intense stimulation (Yuan et al., 2015), similar to what has been observed in synapses (Hosoi et al., 2009; Kawasaki et al., 2011).

\section{Clathrin-Independent Endocytosis}

The recruitment of dynamin to fusion sites can be described by three Gaussian functions (Figure 1A refers to Figure S2 in Yuan et al., 2015). While the last two time constants match that of clathrin recruitment, the first one represents recruitment to sites $\sim 2 \mathrm{~s}$ after a fusion event and independent of clathrin (Figure 1A). Assuming that dynamin 1-dependent endocytosis recycles a vesicle with a size similar to that of a dense-core granule ( $\sim 230 \mathrm{~nm}$ in diameter) the membrane internalized by the clathrin-independent endocytosis shall be much larger than those internalized by the fast, clathrin-dependent pathway, in consistent of membrane capacitance experiments conducted in rat pancreatic $\beta$-cells (He et al., 2008). Such clathrin-independent fast recruitments of dynamin may also profoundly contribute to the fast membrane capacitance decay recorded in synapses and other endocrine cells (Artalejo et al., 2002; Lou et al., 2008; Hosoi et al., 2009). In addition to dynamin, actin also plays an indispensable role in the clathrin-independent endocytosis in pancreatic $\beta$-cells (He et al., 2008).

The identity of clathrin-independent, actin-dependent fast endocytosis is unlikely to be KR in $\beta$-cells, given that exocytosis with fusion pores smaller than $1 \mathrm{~nm}$ lasts $<1 \mathrm{~s}$ in $\beta$-cells (Takahashi et al., 2002). Closure of a large fusion pore formed by KS (cavicapture) is likely to be the corresponding form of the fast clathrin-independent endocytosis. Bulk endocytosis, also found in endocrine cells and synapses (Wen et al., 2012; Watanabe et al., 2013), can be fast and independent of clathrin. Structurally, bulk endocytosis could be the reversal of sequential fusion or multivesicular endocytosis. The main difference between a bulk endocytosis and a cavicapture event is that the quantity of the plasma membrane retrieved by a single endocytic process is larger in the former. To differentiate these possibilities, we must directly visualize the membrane structures of fusion sites on the plasma membrane with imaging techniques. Of course, bulk endocytosis could also be unrelated to sequential exocytosis but related to a continuously invaginated plasma membrane driven by vesicular proteins, lipids, and endocytic machinery.

Finally, a clathrin-independent and dynamin-independent endocytosis is found in calyx neurons (Xu et al., 2008). However, without a definite molecular marker, this endocytic process cannot be studied further. In contrast, an ultrafast, clathrinindependent endocytosis is found in central synapses (Watanabe et al., 2013), which was inhibited by dynasore. However, given that dynasore affects cellular cholesterol, lipid rafts, and actin as well as dynamin (Preta et al., 2015), whether ultrafast endocytosis depends on dynamin remains to be proved. Actin is found to be required for the fast endocytosis in neurons (Delvendahl et al., 2016; Wu et al., 2016; Soykan et al., 2017), similar to what have been demonstrated in endocrine $\beta$-cells (He et al., 2008). However, how this fast endocytosis defined by electrophysiological and fluorescence experiments correlates with ultrafast endocytosis defined by the rapidlyfreezing electron microscopy needs to be explored in the future.

\section{MOLECULAR MECHANISMS FOR EXO-ENDOCYTOSIS COUPLING}

Different factors couple endocytosis with exocytosis, including cytosolic $\mathrm{Ca}^{2+}$, lipids, cytoskeleton and proteins (Wu L. G. et al., 2014). Here, we briefly summarize how they are proposed to function in exo-endocytosis coupling.

\section{$\mathrm{Ca}^{2+}$}

$\mathrm{Ca}^{2+}$ influx through voltage-gated calcium channels triggers exocytosis in excitable cells. Synaptotagmin is the established $\mathrm{Ca}^{2+}$ sensor for triggering vesicle exocytosis. An increase in $\left[\mathrm{Ca}^{2+}\right]_{\mathrm{i}}$, on the other hand, accelerates but does not affect the amplitude of both clathrin-independent and clathrin-dependent endocytosis in $\beta$-cells (He et al., 2008). Not surprisingly, $\left[\mathrm{Ca}^{2+}\right]_{\mathrm{i}}$ elevation is found to initiate all forms of endocytosis (fast endocytosis, slow endocytosis, and bulk endocytosis) in calyx neurons (Hosoi et al., 2009; Wu et al., 2009). Because endocytosis is intimately linked to the prior exocytosis, the impact of $\mathrm{Ca}^{2+}$ influx on endocytosis may be a result of the impact of $\mathrm{Ca}^{2+}$ on membrane additions due to exocytosis. However, the relationship between the speed of endocytosis and $\left[\mathrm{Ca}^{2+}\right]_{\mathrm{i}}$ (He et al., 2008) is different than that between $\left[\mathrm{Ca}^{2+}\right]_{\mathrm{i}}$ and exocytosis (Wan et al., 2004). Similarly, deletion or mutation of both the C2A and C2B domains of the calcium-binding domains of synaptotagmin 1 prolongs the time constant of slow endocytosis by $30-50 \%$ but does not completely block the endocytosis (Yao et al., 2011). These data suggest that $\mathrm{Ca}^{2+}$ may affect the endocytic route via a pathway different from that for exocytosis.

The application of various calmodulin inhibitors blocks all types of endocytosis in calyx neurons, suggesting that calmodulin could be one $\mathrm{Ca}^{2+}$ sensor for endocytosis (Wu et al., 2009). The mechanism by which calmodulin phosphorylation initiates endocytosis remains to be determined. Calcineurin, the phosphatase that dephosphorylates many endocytic proteins (Cousin and Robinson, 2001), could be one main downstream target of calmodulin (Wu X. S. et al., 2014). Calcineurin has been shown to selectively dephosphorylate neuronal specific dynamin 1 and dynamin 3 but not ubiquitous dynamin 2 . Such dephosphorylation is associated with the recruitment of F-BAR protein, syndapin I (Anggono et al., 2006), and may be critical for the stimulation of bulk endocytosis in synapses (Clayton et al., 2009). However, a large number of studies using blockers of calcineurin do not reach a consensus ( $\mathrm{Wu} \mathrm{L}$. G. et al., 2014). Therefore, it is unclear whether such the controversy is due to the different synapses involved or a lack of specificity 
of pharmacological blockers. Resolving this issue is critical for understanding how calcium influx triggers endocytosis.

\section{Lipids}

Phosphatidylinositol 4,5-bisphosphate $\left(\mathrm{PIP}_{2}\right)$ is a minority phospholipid of the inner leaflet of plasma membranes (Suh and Hille, 2008). On the one hand, $\mathrm{PIP}_{2}$ activates voltage gated $\mathrm{Ca}^{2+}$ channels and slows channel rundown, which is upstream of vesicle exocytosis. On the other hand, $\mathrm{PIP}_{2}$ also interacts with a number of proteins essential for the exocytosis machinery, such as syntaxin 1, Munc13, synaptotagmin and Doc2, either via the $\mathrm{C} 2$ domain or via an electrostatic interaction with basic amino acids (Koch and Holt, 2012). PIP 2 binds to syntaxin and Munc13, which regulate the readily releasable pool of vesicles, and the $\mathrm{PIP}_{2}$ :synaptotagmin interaction seems to be essential for the $\mathrm{Ca}^{2+}$-dependent structure changes that catalyze the SNARE assembly. $\mathrm{PIP}_{2}$ also serves as a central hub for the organization of different endocytic proteins. Through electrostatic interactions with dynamin, the adaptor protein 2 (AP2), membrane curvature sensing protein $\mathrm{FCHo}$, amphiphysin, and assessor proteins, such as epsin and synaptojanin, $\mathrm{PIP}_{2}$ facilitates the initiation, assembly, maturation, and final scission of CCPs. Therefore, $\mathrm{PIP}_{2}$, being in the center of recruiting proteins important for exocytosis and endocytosis, could be one crucial coupling factor.

Downstream of both $\mathrm{Ca}^{2+}$ and $\mathrm{PIP}_{2}$, we have shown that diaglycerol (DAG) could be another lipid that coordinates exocytosis and endocytosis. $\mathrm{Ca}^{2+}$ influx activates $\mathrm{Ca}^{2+}$ dependent phospholipase $\mathrm{C}$, which breaks down $\mathrm{PIP}_{2}$ into inositol trisphosphate $\left(\mathrm{IP}_{3}\right)$ and $\mathrm{DAG}$, which is locally enriched around fusion sites in pancreatic $\beta$-cells. In return, DAG binds to Munc13 and activates protein kinase $\mathrm{C}$, both of which are essential to vesicle exocytosis. As a lipid that induces negative membrane curvature, DAG microdomains accumulated at fusion sites reduce the energy of CCP movement on the plasma membrane, thus guiding the movement of preformed CCPs toward fusion sites to mediate fast, clathrin-dependent endocytosis (Yuan et al., 2015).

\section{Cytoskeleton}

Densely packed actin filaments are often seen under the plasma membrane. Actin and related factors, such as Cdc42, N-WASP, and actin binding protein (ABP), interact directly or indirectly with active zone scaffolding proteins such as piccolo, organizing vesicle trafficking to, and fusion at the active zone. Cdc42 and N-WASP also interact with coat proteins of CCPs such as intersectin. These data suggest that actin could act as a bridge between exocytosis and endocytosis (Alabi and Tsien, 2013).

Microtubules, on the other hand, are often believed to bridge between the cell interior to the actin filaments close to the plasma membrane. However, microtubules originated from the Golgi can also touch the plasma membrane by CLASP, a microtubule-associated capping protein (Lansbergen et al., 2006). Through its interaction with LL5 $\beta$, CLASP interacts with ELKS, another active zone scaffolding protein, and helps to anchor dynamic microtubule filaments at fusion sites. We show that a mutation of CLASP inhibits exocytosis in pancreatic $\beta$ cells and reduces coupled endocytosis along with a reduction in the simultaneous movement of CCPs toward the fusion sites (Yuan et al., 2015). Therefore, microtubules organized by CLASP and ELKS may be another factor that couples exocytosis with fast clathrin-dependent endocytosis in secretory cells.

\section{Proteins}

SNARE proteins and associated proteins such as synaptotagmin and Munc13 are essential for exocytosis, while also interacting with proteins critical for endocytosis (Wu L. G. et al., 2014). However, different from their active roles in exocytosis, the roles of SNARE and associated proteins in endocytosis may be providing domains for AP2 and other adaptor proteins to recognize and bind. In this sense, their roles in endocytosis are permissive and non-essential. Dynamin is another protein that may participate critically in both exocytosis and endocytosis. As a GTPase, the role of dynamin in mediating fission of endocytic vesicle is well-known. On the exocytosis side, transfecting PC12 cells with a dynamin mutant with elevated GTPase activity shortened the foot duration of amperometry recordings, while the opposite occurred with the overexpression of a dynamin mutant with reduced GTPase activity (Anantharam et al., 2011; Jackson et al., 2015). These experiments place dynamin at the very beginning of exocytosis regulation, where the fusion pore is smaller than $1 \mathrm{~nm}$. How this function of dynamin is correlated with its impact on the endocytic machinery remains elusive. Accordingly, deletion of dynamin1 impairs both endocytosis and exocytosis at central synapses and produces different synaptic plasticity through distinct mechanisms (Mahapatra et al., 2016; Mahapatra and Lou, 2017); deletion of dynamin-2 in pancreatic $\beta$-cells leads to defects in clathrin-mediated endocytosis and biphasic insulin release (Fan et al., 2015).

\section{SUMMARY AND FUTURE PERSPECTIVES}

We have summarized the above-mentioned mechanisms regarding exocytosis, endocytosis and possible coupling factors in Figure 2. From a macroscopic view, exocytosis may be matched with endocytosis: full fusion with clathrin-mediated endocytosis, KR and KS with clathrin-independent endocytosis, and sequential fusion and multivesicular exocytosis with bulk endocytosis. In this sense, the fate of the components of the fusing vesicle may be pre-determined at the moment of its choice of fusion modes. Therefore, understanding the early fusion intermediates of a vesicle, such as the hemifusion state, pore opening, dilation, and shape retention, will be instrumental for the understanding of the whole coupled process.

The listed classification of different exocytosis and endocytosis subtypes is not based on molecular mechanism but rather hinges on studies that involve different experiments conducted on different cell types. The terminologies defined by different methods may not be mutually inclusive or exclusive. For example, bulk endocytosis is usually regarded as a subcategory of 


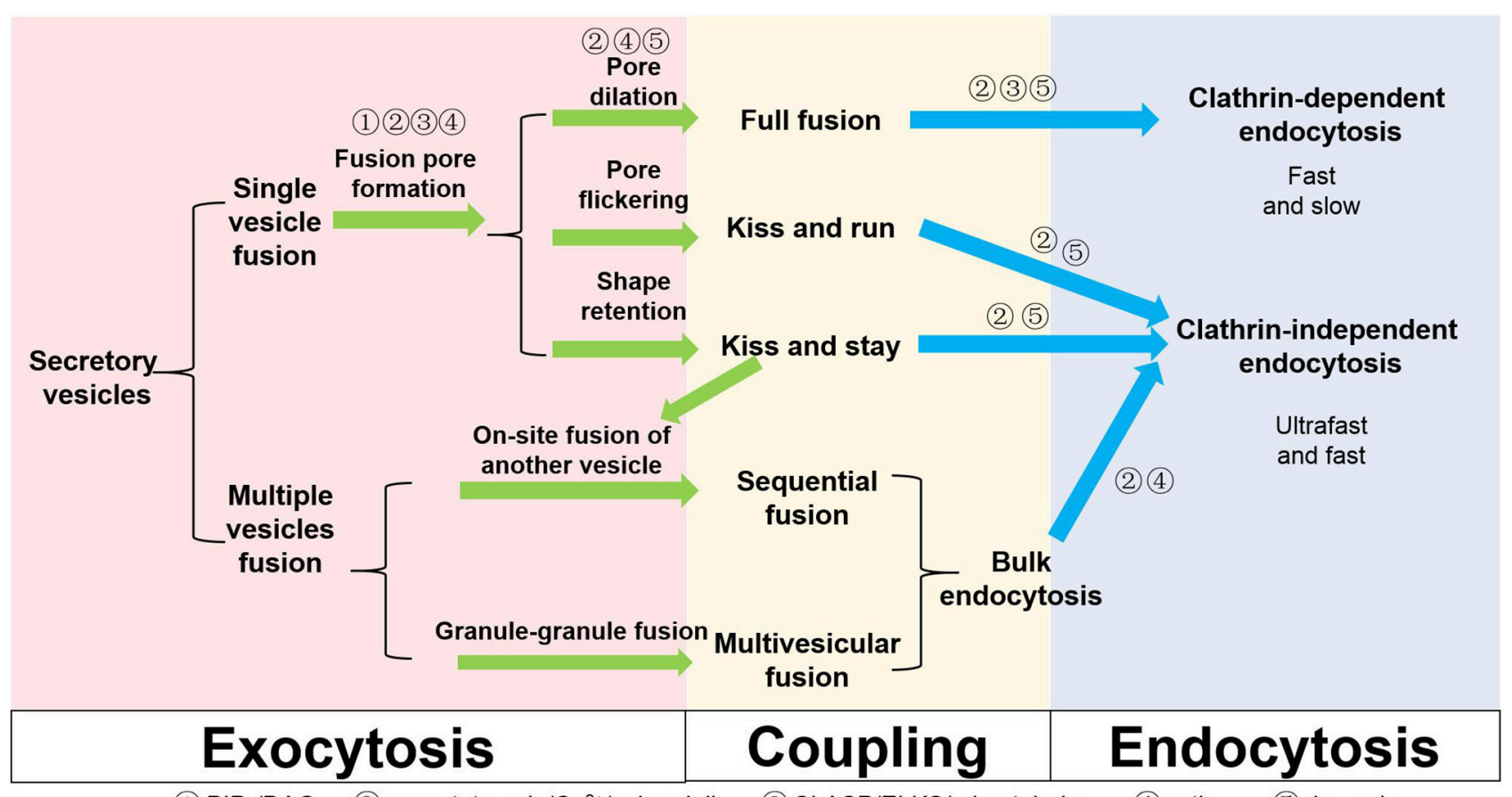

(1) $\mathrm{PIP}_{2} / \mathrm{DAG}$ (2) synaptotagmin/ $\mathrm{Ca}^{2+} /$ calmodulin (3) $\mathrm{CLASP} / \mathrm{ELKS} / \mathrm{microtubules}$ (4) actin (5) dynamin

FIGURE 2 | Different types of exocytosis, endocytosis and coupling factors in secretory cells. Coupling factors and their roles in different steps are also listed on the scheme.

clathrin-independent endocytosis. However, the bulk membrane invaginations observed in secretory cells under EM, which are often taken as evidence supporting bulk endocytosis, may support the internalization of small or large chunks of membrane in a clathrin-dependent manner in live cell studies. KR and KS may be one uniform process at different stages but could also be two distinct processes with non-overlapping mechanisms. To differentiate these controversies, it is important to sort out molecules that are exclusively used for some specific processes, in addition to actin for clathrin-independent endocytosis ( $\mathrm{He}$ et al., 2008; Delvendahl et al., 2016). Alternatively, we shall examine the same process in the same cells using multiple techniques. For example, combining cell-attached membrane capacitance measurements with imaging vesicular lipids in endocrine cells will help clarify whether lipid exchange occurs between the vesicle and the plasma membrane during the flickering of a small fusion pore. Simultaneous imaging of vesicular components and extracellularly applied fluorescent dextran of different sizes will help monitor the dilation of a fusion pore from $\sim 1 \mathrm{~nm}$ to a much larger in diameter (Takahashi et al., 2002). This will differentiate KR and KS and ultimately determine the size of fusion pores accompanying KS exocytosis. Monitoring the shape of the membrane may reveal clues of hemifusion in live cells (Zhao et al., 2016) and will also confirm or disapprove the compound fusion/multivesicular exocytosis theories and their physiological significance. Finally, operating at a nanometer scale with lifetimes of milliseconds, most of the fusion intermediate structures described here can hardly be directly discerned even with state-of-the-art superresolution microscopy methodologies (Huang et al., 2009; Schermelleh et al., 2010). Despite differences in exocytosis kinetics and the organization of fusion sites between synapses and endocrine cells, we believe that the core exo-endocytosis coupling mechanism is conserved. Therefore, if we can improve the temporal and spatial resolution and duration of current super-resolution imaging technologies, direct visualization of fusion pore intermediates in endocrine cells may invoke new insights that would render much of the discussed theories here obsolete.

\section{AUTHOR CONTRIBUTIONS}

All authors listed, have made substantial, direct and intellectual contribution to the work, and approved it for publication.

\section{ACKNOWLEDGMENTS}

The work was supported by grants from the National Natural Science Foundation of China (31327901, 31428004, 31521062, 31570839), the Major State Basic Research Program of China (2013CB531200), the National Science and Technology Major Project Program (2016YFA0500400), the Beijing Natural Science Foundation (7142071), and the Beijing Health System High Level Health Technical Personnel (2014-3-058). 


\section{REFERENCES}

Alabi, A. A., and Tsien, R. W. (2013). Perspectives on kiss-and-run: role in exocytosis, endocytosis, and neurotransmission. Annu. Rev. Physiol. 75, 393-422. doi: 10.1146/annurev-physiol-020911-153305

Albillos, A., Dernick, G., Horstmann, H., Almers, W., Alvarez de Toledo, G., and Lindau, M. (1997). The exocytotic event in chromaffin cells revealed by patch amperometry. Nature 389, 509-512. doi: 10.1038/39081

Ales, E., Tabares, L., Poyato, J. M., Valero, V., Lindau, M., and Alvarez de Toledo, G. (1999). High calcium concentrations shift the mode of exocytosis to the kiss-and-run mechanism. Nat. Cell Biol. 1, 40-44.

An, S., and Zenisek, D. (2004). Regulation of exocytosis in neurons and neuroendocrine cells. Curr. Opin. Neurobiol. 14, 522-530. doi: 10.1016/j.conb. 2004.08.008

Anantharam, A., Bittner, M. A., Aikman, R. L., Stuenkel, E. L., Schmid, S. L., Axelrod, D., et al. (2011). A new role for the dynamin GTPase in the regulation of fusion pore expansion. Mol. Biol. Cell 22, 1907-1918. doi: 10.1091/mbc.E11-02-0101

Anggono, V., Smillie, K. J., Graham, M. E., Valova, V. A., Cousin, M. A., and Robinson, P. J. (2006). Syndapin I is the phosphorylation-regulated dynamin I partner in synaptic vesicle endocytosis. Nat. Neurosci. 9, 752-760. doi: $10.1038 / \mathrm{nn} 1695$

Aoki, R., Kitaguchi, T., Oya, M., Yanagihara, Y., Sato, M., Miyawaki, A., et al. (2010). Duration of fusion pore opening and the amount of hormone released are regulated by myosin II during kiss-and-run exocytosis. Biochem. J. 429, 497-504. doi: 10.1042/BJ20091839

Artalejo, C. R., Elhamdani, A., and Palfrey, H. C. (2002). Sustained stimulation shifts the mechanism of endocytosis from dynamin-1dependent rapid endocytosis to clathrin- and dynamin-2-mediated slow endocytosis in chromaffin cells. Proc. Natl. Acad. Sci. U.S.A. 99, 6358-6363. doi: 10.1073/pnas.082658499

Bao, H., Goldschen-Ohm, M., Jeggle, P., Chanda, B., Edwardson, J. M., and Chapman, E. R. (2016). Exocytotic fusion pores are composed of both lipids and proteins. Nat. Struct. Mol. Biol. 23, 67-73. doi: 10.1038/nsmb.3141

Barg, S., Knowles, M. K., Chen, X., Midorikawa, M., and Almers, W. (2010). Syntaxin clusters assemble reversibly at sites of secretory granules in live cells. Proc. Natl. Acad. Sci. U.S.A. 107, 20804-20809. doi: 10.1073/pnas.10148 23107

Breckenridge, L. J., and Almers, W. (1987). Currents through the fusion pore that forms during exocytosis of a secretory vesicle. Nature 328, 814-817. doi: $10.1038 / 328814 \mathrm{a} 0$

Cano, R., and Tabares, L. (2016). The active and periactive zone organization and the functional properties of small and large synapses. Front. Synaptic Neurosci. 8:12. doi: $10.3389 /$ fnsyn.2016.00012

Ceccarelli, B., Hurlbut, W. P., and Mauro, A. (1973). Turnover of transmitter and synaptic vesicles at the frog neuromuscular junction. J. Cell Biol. 57, 499-524. doi: $10.1083 /$ jcb.57.2.499

Chanturiya, A., Chernomordik, L. V., and Zimmerberg, J. (1997). Flickering fusion pores comparable with initial exocytotic pores occur in proteinfree phospholipid bilayers. Proc. Natl. Acad. Sci. U.S.A. 94, 14423-14428. doi: 10.1073/pnas.94.26.14423

Clayton, E. L., Anggono, V., Smillie, K. J., Chau, N., Robinson, P. J., and Cousin, M. A. (2009). The phospho-dependent dynamin-syndapin interaction triggers activity-dependent bulk endocytosis of synaptic vesicles. J. Neurosci. 29, 7706-7717. doi: 10.1523/JNEUROSCI.1976-09.2009

Cousin, M. A., and Robinson, P. J. (2001). The dephosphins: dephosphorylation by calcineurin triggers synaptic vesicle endocytosis. Trends Neurosci. 24, 659-665. doi: 10.1016/S0166-2236(00)01930-5

Delvendahl, I., Vyleta, N. P., von Gersdorff, H., and Hallermann, S. (2016). Fast, temperature-sensitive and clathrin-independent endocytosis at central synapses. Neuron 90, 492-498. doi: 10.1016/j.neuron.2016.03.013

Dhara, M., Yarzagaray, A., Schwarz, Y., Dutta, S., Grabner, C., Moghadam, P. K., et al. (2014). Complexin synchronizes primed vesicle exocytosis and regulates fusion pore dynamics. J. Cell Biol. 204, 1123-1140. doi: 10.1083/jcb.201311085

Domanska, M. K., Kiessling, V., Stein, A., Fasshauer, D., and Tamm, L. K. (2009). Single vesicle millisecond fusion kinetics reveals number of SNARE complexes optimal for fast SNARE-mediated membrane fusion. J. Biol. Chem. 284, 32158-32166. doi: 10.1074/jbc.M109.047381
Fan, F., Ji, C., Wu, Y., Ferguson, S. M., Tamarina, N., Philipson, L. H., et al. (2015). Dynamin 2 regulates biphasic insulin secretion and plasma glucose homeostasis. J. Clin. Invest. 125, 4026-4041. doi: 10.1172/JCI80652

Fang, Q., Berberian, K., Gong, L. W., Hafez, I., Sorensen, J. B., and Lindau, M. (2008). The role of the C terminus of the SNARE protein SNAP-25 in fusion pore opening and a model for fusion pore mechanics. Proc. Natl. Acad. Sci. U.S.A. 105, 15388-15392. doi: 10.1073/pnas.0805377105

Granseth, B., Odermatt, B., Royle, S. J., and Lagnado, L. (2006). Clathrin-mediated endocytosis is the dominant mechanism of vesicle retrieval at hippocampal synapses. Neuron 51, 773-786. doi: 10.1016/j.neuron.2006.08.029

Gucek, A., Jorgacevski, J., Singh, P., Geisler, C., Lisjak, M., Vardjan, N., et al. (2016). Dominant negative SNARE peptides stabilize the fusion pore in a narrow, release-unproductive state. Cell. Mol. Life Sci. 73, 3719-3731. doi: 10.1007/s00018-016-2213-2

Han, X., Wang, C. T., Bai, J., Chapman, E. R., and Jackson, M. B. (2004) Transmembrane segments of syntaxin line the fusion pore of $\mathrm{Ca}^{2+}$-triggered exocytosis. Science 304, 289-292. doi: 10.1126/science.1095801

He, L., Wu, X. S., Mohan, R., and Wu, L. G. (2006). Two modes of fusion pore opening revealed by cell-attached recordings at a synapse. Nature 444, 102-105. doi: $10.1038 /$ nature 05250

He, L., Xue, L., Xu, J., McNeil, B. D., Bai, L., Melicoff, E., et al. (2009). Compound vesicle fusion increases quantal size and potentiates synaptic transmission. Nature 459, 93-97. doi: 10.1038/nature07860

He, Z., Fan, J., Kang, L., Lu, J., Xue, Y., Xu, P., et al. (2008). $\mathrm{Ca}^{2+}$ triggers a novel clathrin-independent but actin-dependent fast endocytosis in pancreatic beta cells. Traffic 9, 910-923. doi: 10.1111/j.1600-0854.2008.00730.x

Heuser, J. E., and Reese, T. S. (1981). Structural changes after transmitter release at the frog neuromuscular junction. J. Cell Biol. 88, 564-580. doi: $10.1083 /$ jcb.88.3.564

Holroyd, P., Lang, T., Wenzel, D., De Camilli, P., and Jahn, R. (2002). Imaging direct, dynamin-dependent recapture of fusing secretory granules on plasma membrane lawns from PC12 cells. Proc. Natl. Acad. Sci. U.S.A. 99, 16806-16811. doi: 10.1073/pnas.222677399

Hosoi, N., Holt, M., and Sakaba, T. (2009). Calcium dependence of exoand endocytotic coupling at a glutamatergic synapse. Neuron 63, 216-229. doi: 10.1016/j.neuron.2009.06.010

Huang, B., Bates, M., and Zhuang, X. (2009). Super-resolution fluorescence microscopy. Annu. Rev. Biochem. 78, 993-1016. doi: 10.1146/annurev.biochem. 77.061906.092014

Jackson, J., Papadopulos, A., Meunier, F. A., McCluskey, A., Robinson, P. J., and Keating, D. J. (2015). Small molecules demonstrate the role of dynamin as a bi-directional regulator of the exocytosis fusion pore and vesicle release. Mol. Psychiatry 20, 810-819. doi: 10.1038/mp.2015.56

Kawasaki, F., Iyer, J., Posey, L. L., Sun, C. E., Mammen, S. E., Yan, H., et al. (2011). The DISABLED protein functions in CLATHRIN-mediated synaptic vesicle endocytosis and exoendocytic coupling at the active zone. Proc. Natl. Acad. Sci. U.S.A. 108, E222-E229. doi: 10.1073/pnas.1102231108

Kishimoto, T., Liu, T. T., Hatakeyama, H., Nemoto, T., Takahashi, N., and Kasai, H. (2005). Sequential compound exocytosis of large densecore vesicles in PC12 cells studied with TEPIQ (two-photon extracellular polar-tracer imaging-based quantification) analysis. J. Physiol. 568, 905-915. doi: 10.1113/jphysiol.2005.094003

Koch, M., and Holt, M. (2012). Coupling exo- and endocytosis: an essential role for PIP(2) at the synapse. Biochim. Biophys. Acta 1821, 1114-1132. doi: 10.1016/j.bbalip.2012.02.008

Kwan, E. P., and Gaisano, H. Y. (2005). Glucagon-like peptide 1 regulates sequential and compound exocytosis in pancreatic islet beta-cells. Diabetes 54, 2734-2743. doi: 10.2337/diabetes.54.9.2734

Lai, Y., Diao, J., Liu, Y., Ishitsuka, Y., Su, Z., Schulten, K., et al. (2013). Fusion pore formation and expansion induced by $\mathrm{Ca}^{2+}$ and synaptotagmin 1. Proc. Natl. Acad. Sci. U.S.A. 110, 1333-1338. doi: 10.1073/pnas.1218818110

Lansbergen, G., Grigoriev, I., Mimori-Kiyosue, Y., Ohtsuka, T., Higa, S., Kitajima, I., et al. (2006). CLASPs attach microtubule plus ends to the cell cortex through a complex with LL5beta. Dev. Cell 11, 21-32. doi: 10.1016/j.devcel.2006.05.012

Lindau, M., and Alvarez de Toledo, G. (2003). The fusion pore. Biochim. Biophys. Acta 1641, 167-173. doi: 10.1016/S0167-4889(03)00085-5

Lou, X., Paradise, S., Ferguson, S. M., and De Camilli, P. (2008). Selective saturation of slow endocytosis at a giant glutamatergic central synapse lacking dynamin 1 . 
Proc. Natl. Acad. Sci. U.S.A. 105, 17555-17560. doi: 10.1073/pnas.08096 21105

MacDonald, P. E., Braun, M., Galvanovskis, J., and Rorsman, P. (2006). Release of small transmitters through kiss-and-run fusion pores in rat pancreatic beta cells. Cell Metab. 4, 283-290. doi: 10.1016/j.cmet.2006.08.011

Mahapatra, S., and Lou, X. (2017). Dynamin-1 deletion enhances post-tetanic potentiation and quantal size after tetanic stimulation at the calyx of Held. J. Physiol. 595, 193-206. doi: 10.1113/JP271937

Mahapatra, S., Fan, F., and Lou, X. (2016). Tissue-specific dynamin-1 deletion at the calyx of Held decreases short-term depression through a mechanism distinct from vesicle resupply. Proc. Natl. Acad. Sci. U.S.A. 113, E3150-E3158. doi: 10.1073/pnas.1520937113

Mohrmann, R., de Wit, H., Verhage, M., Neher, E., and Sorensen, J. B. (2010). Fast vesicle fusion in living cells requires at least three SNARE complexes. Science 330, 502-505. doi: 10.1126/science.1193134

Ornberg, R. L., and Reese, T. S. (1981). Beginning of exocytosis captured by rapidfreezing of Limulus amebocytes. J. Cell Biol. 90, 40-54. doi: 10.1083/jcb.90.1.40

Pickett, J. A., and Edwardson, J. M. (2006). Compound exocytosis: mechanisms and functional significance. Traffic 7, 109-116. doi: 10.1111/j.1600-0854. 2005.00372.x

Preta, G., Cronin, J. G., and Sheldon, I. M. (2015). Dynasore - not just a dynamin inhibitor. Cell Commun. Signal. 13, 24. doi: 10.1186/s12964-015-0102-1

Reigada, D., Diez-Perez, I., Gorostiza, P., Verdaguer, A., Gomez de Aranda, I., Pineda, O., et al. (2003). Control of neurotransmitter release by an internal gel matrix in synaptic vesicles. Proc. Natl. Acad. Sci. U.S.A. 100, 3485-3490. doi: $10.1073 /$ pnas. 0336914100

Rizzoli, S. O., and Jahn, R. (2007). Kiss-and-run, collapse and 'readily retrievable' vesicles. Traffic 8, 1137-1144. doi: 10.1111/j.1600-0854.2007.00614.x

Schermelleh, L., Heintzmann, R., and Leonhardt, H. (2010). A guide to super-resolution fluorescence microscopy. J. Cell Biol. 190, 165-175. doi: $10.1083 /$ jcb. 201002018

Sharma, S., and Lindau, M. (2016). The mystery of the fusion pore. Nat. Struct. Mol. Biol. 23, 5-6. doi: 10.1038/nsmb.3157

Sombers, L. A., Hanchar, H. J., Colliver, T. L., Wittenberg, N., Cans, A., Arbault, S., et al. (2004). The effects of vesicular volume on secretion through the fusion pore in exocytotic release from PC12 cells. J. Neurosci. 24, 303-309. doi: 10.1523/JNEUROSCI.1119-03.2004

Soykan, T., Kaempf, N., Sakaba, T., Vollweiter, D., Goerdeler, F., Puchkov, D., et al. (2017). Synaptic vesicle endocytosis occurs on multiple timescales and is mediated by formin-dependent actin assembly. Neuron 93, 854-866.e4. doi: 10.1016/j.neuron.2017.02.011

Suh, B. C., and Hille, B. (2008). PIP2 is a necessary cofactor for ion channel function: how and why? Annu. Rev. Biophys. 37, 175-195. doi: 10.1146/annurev.biophys.37.032807.125859

Takahashi, N., Hatakeyama, H., Okado, H., Miwa, A., Kishimoto, T., Kojima, T., et al. (2004). Sequential exocytosis of insulin granules is associated with redistribution of SNAP25. J. Cell Biol. 165, 255-262. doi: 10.1083/jcb.2003 12033

Takahashi, N., Kishimoto, T., Nemoto, T., Kadowaki, T., and Kasai, H. (2002). Fusion pore dynamics and insulin granule exocytosis in the pancreatic islet. Science 297, 1349-1352. doi: 10.1126/science.1073806

Taraska, J. W., and Almers, W. (2004). Bilayers merge even when exocytosis is transient. Proc. Natl. Acad. Sci. U.S.A. 101, 8780-8785. doi: 10.1073/pnas.0401316101

Taraska, J. W., Perrais, D., Ohara-Imaizumi, M., Nagamatsu, S., and Almers, W. (2003). Secretory granules are recaptured largely intact after stimulated exocytosis in cultured endocrine cells. Proc. Natl. Acad. Sci. U.S.A. 100, 2070-2075. doi: 10.1073/pnas.0337526100

Thorn, P., and Gaisano, H. (2012). Molecular control of compound exocytosis: a key role for VAMP8. Commun. Integr. Biol. 5, 61-63. doi: 10.4161/cib.18058

Tsuboi, T., and Rutter, G. A. (2003). Multiple forms of "kiss-and-run" exocytosis revealed by evanescent wave microscopy. Curr. Biol. 13, 563-567. doi: 10.1016/S0960-9822(03)00176-3

Tsuboi, T., McMahon, H. T., and Rutter, G. A. (2004). Mechanisms of dense core vesicle recapture following "kiss and run" ("cavicapture") exocytosis in insulin-secreting cells. J. Biol. Chem. 279, 47115-47124. doi: 10.1074/jbc.M4081 79200

van den Bogaart, G., Holt, M. G., Bunt, G., Riedel, D., Wouters, F. S., and Jahn, R. (2010). One SNARE complex is sufficient for membrane fusion. Nat. Struct. Mol. Biol. 17, 358-364. doi: 10.1038/nsmb.1748 van den Bogaart, G., Meyenberg, K., Risselada, H. J., Amin, H., Willig, K. I., Hubrich, B. E., et al. (2011). Membrane protein sequestering by ionic proteinlipid interactions. Nature 479, 552-555. doi: 10.1038/nature10545

Wan, Q. F., Dong, Y., Yang, H., Lou, X., Ding, J., and Xu, T. (2004). Protein kinase activation increases insulin secretion by sensitizing the secretory machinery to $\mathrm{Ca}^{2+}$. J. Gen. Physiol. 124, 653-662. doi: 10.1085/jgp.200409082

Wang, C. T., Grishanin, R., Earles, C. A., Chang, P. Y., Martin, T. F., Chapman, E. R., et al. (2001). Synaptotagmin modulation of fusion pore kinetics in regulated exocytosis of dense-core vesicles. Science 294, 1111-1115. doi: 10.1126/science.1064002

Watanabe, S., Rost, B. R., Camacho-Perez, M., Davis, M. W., Sohl-Kielczynski, B., Rosenmund, C., et al. (2013). Ultrafast endocytosis at mouse hippocampal synapses. Nature 504, 242-247. doi: 10.1038/nature12809

Weiss, A. N., Anantharam, A., Bittner, M. A., Axelrod, D., and Holz, R. W. (2014). Lumenal protein within secretory granules affects fusion pore expansion. Biophys. J. 107, 26-33. doi: 10.1016/j.bpj.2014.04.064

Wen, D., Xue, Y., Liang, K., Yuan, T., Lu, J., Zhao, W., et al. (2012). Bulk-like endocytosis plays an important role in the recycling of insulin granules in pancreatic beta cells. Protein Cell 3, 618-626. doi: 10.1007/s13238-012-2938-0

Wienisch, M., and Klingauf, J. (2006). Vesicular proteins exocytosed and subsequently retrieved by compensatory endocytosis are nonidentical. Nat. Neurosci. 9, 1019-1027. doi: 10.1038/nn1739

Wu, L. G., Hamid, E., Shin, W., and Chiang, H. C. (2014). Exocytosis and endocytosis: modes, functions, and coupling mechanisms. Annu. Rev. Physiol. 76, 301-331. doi: 10.1146/annurev-physiol-021113-170305

$\mathrm{Wu}, \mathrm{W}$., and Wu, L. G. (2007). Rapid bulk endocytosis and its kinetics of fission pore closure at a central synapse. Proc. Natl. Acad. Sci. U.S.A. 104, 10234-10239. doi: 10.1073/pnas.0611512104

Wu, X. S., Lee, S. H., Sheng, J., Zhang, Z., Zhao, W. D., Wang, D., et al. (2016). Actin is crucial for all kinetically distinguishable forms of endocytosis at synapses. Neuron 92, 1020-1035. doi: 10.1016/j.neuron.2016.10.014

Wu, X. S., McNeil, B. D., Xu, J., Fan, J., Xue, L., Melicoff, E., et al. (2009). $\mathrm{Ca}^{2+}$ and calmodulin initiate all forms of endocytosis during depolarization at a nerve terminal. Nat. Neurosci. 12, 1003-1010. doi: 10.1038/nn.2355

Wu, X. S., Zhang, Z., Zhao, W. D., Wang, D., Luo, F., and Wu, L. G. (2014). Calcineurin is universally involved in vesicle endocytosis at neuronal and nonneuronal secretory cells. Cell Rep. 7, 982-988. doi: 10.1016/j.celrep.2014.04.020

Xu, J., McNeil, B., Wu, W., Nees, D., Bai, L., and Wu, L. G. (2008). GTPindependent rapid and slow endocytosis at a central synapse. Nat. Neurosci. 11, 45-53. doi: 10.1038/nn2021

Yao, J., Kwon, S. E., Gaffaney, J. D., Dunning, F. M., and Chapman, E. R. (2011). Uncoupling the roles of synaptotagmin I during endo- and exocytosis of synaptic vesicles. Nat. Neurosci. 15, 243-249. doi: 10.1038/nn.3013

Yuan, T., Liu, L., Zhang, Y., Wei, L., Zhao, S., Zheng, X., et al. (2015). Diacylglycerol guides the hopping of clathrin-coated pits along microtubules for exoendocytosis coupling. Dev. Cell 35, 120-130. doi: 10.1016/j.devcel.2015.09.004

Zhang, Q., Li, Y., and Tsien, R. W. (2009). The dynamic control of kiss-and-run and vesicular reuse probed with single nanoparticles. Science 323, 1448-1453. doi: $10.1126 /$ science. 1167373

Zhao, W. D., Hamid, E., Shin, W., Wen, P. J., Krystofiak, E. S., Villarreal, S. A., et al. (2016). Hemi-fused structure mediates and controls fusion and fission in live cells. Nature 534, 548-552. doi: 10.1038/nature18598

Zhou, Z., and Misler, S. (1996). Amperometric detection of quantal secretion from patch-clamped rat pancreatic beta-cells. J. Biol. Chem. 271, 270-277. doi: $10.1074 / j b c .271 .1 .270$

Zhu, Y., Xu, J., and Heinemann, S. F. (2009). Two pathways of synaptic vesicle retrieval revealed by single-vesicle imaging. Neuron 61, 397-411. doi: 10.1016/j.neuron.2008.12.024

Conflict of Interest Statement: The authors declare that the research was conducted in the absence of any commercial or financial relationships that could be construed as a potential conflict of interest.

Copyright $\odot 2017$ Liang, Wei and Chen. This is an open-access article distributed under the terms of the Creative Commons Attribution License (CC BY). The use, distribution or reproduction in other forums is permitted, provided the original author(s) or licensor are credited and that the original publication in this journal is cited, in accordance with accepted academic practice. No use, distribution or reproduction is permitted which does not comply with these terms. 